Check for updates

Cite this: Mater. Adv., 2021, 2,5723

Received 30th March 2021, Accepted 25th July 2021

DOI: $10.1039 / \mathrm{d} 1 \mathrm{ma} 00286 \mathrm{~d}$

rsc.li/materials-advances

\section{Synthesis and characterization of $\beta$-cyclodextrin/ carboxymethyl chitosan/hydroxyapatite fused with date seed extract nanocomposite scaffolds for regenerative bone tissue engineering $\dagger$}

\author{
Reshma Jolly, ${ }^{a}$ Mohammad Furkan, ${ }^{b}$ Aijaz Ahmed Khan, ${ }^{c}$ Syed Sayeed Ahmed, ${ }^{d}$ \\ Sharique Alam, ${ }^{e}$ Mohd Ahmadullah Farooqi, ${ }^{f}$ Rizwan Hasan Khan ${ }^{\mathrm{b}}$ and \\ Mohammad Shakir (iD *a
}

\begin{abstract}
Herein, for the first time, heterojunctions containing nano-hydroxyapatite/carboxymethyl chitosan/ $\beta$-cyclodextrin fused with date seed extract (DSE) were synthesized at different concentrations using co-precipitation to generate $\mathrm{BCHD}, \mathrm{BCHD} 2$ and $\mathrm{BCHD} 3$ nanocomposite scaffolds with tunable size and amendable surface properties. FTIR, XRD and mechanical analysis studies confirmed the presence of different constituents in the proposed nanocomposite scaffolds displaying strong interactions with an optimal compressive modulus shown by BCHD3 $[1533 \pm 2.69 \mathrm{MPa}]$, relative to nano-hydroxyapatite/ carboxymethyl chitosan $(\mathrm{CH})$ nanocomposite scaffolds. The results of TEM, water contact angle and SEM revealed the homogenous distribution of needle-shaped particles having an average size ranging between 18 and $25 \mathrm{~nm}$ with a moderate hydrophilicity [WCA $=42.5^{\circ}$ ] and an interconnected rough morphology. Furthermore, the BCHD nanocomposite scaffolds were investigated for their biomimetic mineralization ability using simulated body fluid by mimicking the inorganic composition of human blood plasma, which demonstrated superior hydroxyapatite nucleation. The BCHD3 nanocomposite scaffolds showed an advanced protein adsorption $\left[130 \pm 41 \mu \mathrm{g} \mathrm{cm}{ }^{-2}\right]$ and active alkaline phosphatase performance and did not exacerbate lactate dehydrogenase leakage of MG63 osteoblast-like cells relative to $\mathrm{CH}$. The comprehensive in vivo study identified better bone regeneration in the repair of critical size calvarial defects $[8 \mathrm{~mm}]$ in albino rats upon treatment with BCHD3, which is selectively tested as a bare implant in comparison to cerabone, as corroborated by the histopathological and radiological investigations with an average GBD of $94.5 \pm 1.24 \%$. Therefore, the above-mentioned results have shed more light on establishing the efficiency of $\mathrm{BCHD} 3$ to find applications in osteogenic tissue engineering.
\end{abstract}

${ }^{a}$ Inorganic Chemistry Laboratory, Department of Chemistry, AMU, Aligarh 202002, India. E-mail: shakir078@yahoo.com; Tel: +91-9837430035

${ }^{b}$ Interdisciplinary Biotechnology Unit, AMU, Aligarh 202002, India

${ }^{c}$ Neuroanatomy Laboratory, Department of Anatomy, J. N. Medical College, AMU, Aligarh, 202002, India

${ }^{d}$ Department of Oral and Maxillofacial Surgery, Dr. Ziauddin Ahmad Dental College, AMU, Aligarh, 202002, India

${ }^{e}$ Department of Conservative Dentistry \& Endodontics, Dr. Ziauddin Ahmad Dental College, AMU, Aligarh, 202002, India

${ }^{f}$ Department of Building Engineering, College of Architecture and Planning, Imam Abdulrahman Bin Faisal Universtiy, Dammam, 34221, Saudi Arabia $\dagger$ Electronic supplementary information (ESI) available: Materials and methods, characterization, and physicochemical characterization (Fourier transform infrared [FTIR] spectroscopy, X-ray diffraction, mechanical properties, transmission electron microscopy [TEM], and scanning electron microscopy [SEM] with energy dispersive X-ray spectroscopy [EDAX], water contact angle, in vitro calcification assay, protein adsorption, cell proliferation assay, and lactate dehydrogenase (LDH) assay). See DOI: 10.1039/d1ma00286d

\section{Introduction}

Tissue engineering is intended to develop analogues that could sustain, repair, mimic or substitute a biological tissue. ${ }^{1}$ The process of bone regeneration through engineering the tissue is an intricate biological event which involves scaffolds and processes such as vascularisation, cellular and signaling cues, along with intracellular interactions. In this milieu, the principle extracellular matrix (ECM) like micro-environment is provided by biocompatible materials which facilitate the intracellular interactions. There are many properties that should be satisfied by a tissue engineered matrix, which include appropriate mechanical strength, optimal porosity and surface chemistry to name a few. ${ }^{2}$ However, as the process of regeneration of bone inside the scaffolds is complex, the synchronized processes such as osteogenesis and angiogenesis which are involved in bone 
regeneration have often been examined piecemeal using several in vitro and in vivo experimental studies. ${ }^{3}$ Compared to the existing implants, modern strategies, including nanotechnology, ${ }^{4}$ have opened new horizons to reproduce superior structural properties by fabricating materials which could imitate the nano-scaled architecture of the natural bone. For example, nano-crystalline ceramics such as calcium phosphates ${ }^{5}$ have been shown to fuel the bone cell functions causing rapid degradation relative to microcrystalline calcium phosphate.

Recently, the use of nanohydroxyapatite (NHA) as a biomaterial has escalated great attention in the engineering of bone tissues, being the main component of bone. It acts as a template for the mineral phase deposition and triggers new bone formation. ${ }^{6}$ However, the antiresorptive and anabolic ability of NHA are found to be unsatisfactory and its matrices are reported to be brittle and thus, a new strategy is still desired for the development of biocompatible, biodegradable and bioactive scaffolds. To meet this requirement, biopolymers have enthralled researchers because of their inherent properties such as abundance, low cost, extracellular matrix (ECM)-like characteristics appropriate for cell function and survival, biocompatibility, biodegradability and non-toxicity and thus have been the emerging candidates of choice in recent years. ${ }^{7}$ Among the biopolymers, chitosan, the deacetylated form of chitin derived from the exoskeleton of crustaceans, is actually a polyheterosaccharide containing glucosamine and $\mathrm{N}$-acetyl glucosamine units bonded by 1-4 glucosidic linkages and has gained much attention in the field of bone tissue engineering. ${ }^{8}$ Nevertheless, it is associated with disadvantages like low solubility, less load bearing potency and high percentage of degradation that have restricted its utilization. ${ }^{9}$ In order to cope with these limitations, carboxymethyl chitosan ${ }^{10}$ (CMC), a well warranted chitosan-derived polymer, derived from the carboxylation reaction of amine group substitution by smaller alkyl groups, has engrossed great interest in a wide spectrum of biomedical applications, being biocompatible and cost-effective, and owing to the suitable degradation rate. In addition, CMC is better soluble in water in comparison with chitosan without the aid of acid substances, preventing damage to the $\mathrm{pH}$ sensitive antibacterial activity of a drug. ${ }^{11,12}$ At this junction, it is relevant to mention that combining chitosan derivatives with other naturally derived moieties may result in the development of nanocomposite scaffolds with the advantages of enhanced performance in terms of solubilization and permeability. One such structural residue is the class of cyclodextrins, ${ }^{13}$ which are the cyclic oligosaccharides, consisting of 6,7 or $8 \alpha$-D-glucopyranose units, forming toroidalshaped molecules with a hydrophilic exterior and a hydrophobic interior formed by the carbon backbones of glucopyranose monomers, appropriate for the formation of inclusion complexes. Being oligosaccharides, these molecules bear hydroxyl groups that may possibly be employed for further functionalization. ${ }^{14}$ The $\beta$-cyclodextrin ( $\beta$-CD), among cyclodextrins, is acknowledged as a performance accelerator in tissue engineering ${ }^{15}$ by various routes such as (1) sequestering growth factors, (2) augmenting oxygen concentrations in the tissue engineered constructs, and (3) regulating collagen self-assembly. ${ }^{16-18}$ Therefore, these co-polymeric systems possess the cumulative complimentary properties of their constituent polymers. With regard to date seed added values, Phoenix dactylifera L. (date palm), from the Arecaceae family, has recently been studied for its bone regeneration properties. ${ }^{19}$ There are various studies that investigated date seed powder as an economical and major source of antioxidants and fibers due to the attendance of valuable dietary components in addition to the presence of flavones, polyphenols and vitamins (particularly vitamins $\mathrm{C}$ and $\mathrm{E}$ ), which make them function as anti-atherogenic. ${ }^{20}$ In addition, their chemical makeup and phytochemical profile demonstrated in vitro bioactivities and showed applications such as expectorant, hypoglycaemic, aphrodisiac, and antidiarrheic. In addition, the recent study represented that the extract of the date seeds plays a cerebroprotective role in male rats. ${ }^{21}$ Therefore, in view of their valuable essential features, we used date seed extracts with other polymeric counterparts in the present work. The reason to recruit these established biocompatible ${ }^{22}$ components has come from their wide usage in biomedical and pharmaceutical applications due to the associated low cost, biocompatibility and biodegradability. ${ }^{23,24}$

To our knowledge, heterojunctions containing $\beta$-cyclodextrin (B), carboxymethyl chitosan (C), nano-hydroxyapatite (H), and date seed extracts (D) synthesized by co-precipitation to yield the BCHD nanocomposite scaffolds have not been achieved to date. Therefore, the present study was anticipated to provide a favourable combination of chemical, mechanical and biological properties to evaluate the performance of date seed extracts incorporated at different concentrations into a nanohydroxyapatite/carboxymethyl chitosan/ $\beta$-cyclodextrin matrix and its potency was investigated as a competent and efficacious bone implant. The physicochemical characteristics were evaluated in terms of size, shape, crystallinity, morphology and mechanical efficiency. Furthermore, we have also delved into cell viability, ALP, protein adsorption and LDH analysis to study the biocompatible nature of the proposed nanocomposite scaffolds.

\section{Materials and methods}

\subsection{Materials}

3-(4,5-Dimethylthiazol-2-yl)-2,5-diphenyl tetrazolium bromide) (MTT), carboxymethyl chitosan (CMC) and $\beta$-cyclodextrin ( $\beta$-CD) were obtained from Sigma-Aldrich (USA). The other chemicals/ reagents used in the present study are listed in the ESI $\dagger$ under Section S.2.1.

\subsection{Experimental}

2.2.1. Preparation of NHA. Hydroxyapatite nanoparticles (NHA) were synthesized by chemical precipitation as reported in our previous studies. ${ }^{19,25}$ The so obtained product was subjected to ball milling to decrease the average size of the particles for the formation of crystalline NHA.

2.2.2. Preparation of date palm seed extract (DSE). The Ajwa date seeds were manually separated from the fruit and kept in water for 4-5 hours. Subsequently, the soaked seeds 
were washed repeatedly to completely remove any adhered flesh. Then, the seeds were kept for air-drying followed by drying in an oven for $48 \mathrm{~h}$ at $55-60{ }^{\circ} \mathrm{C}$ and milled to form a fine powder with the help of a heavy duty grinder. ${ }^{26}$ To this powder of dried pits, distilled water was added to make the mixture of $50 \mathrm{gm} \mathrm{L}^{-1}$ which was boiled until it turned brownish and then filtered and stored at $4{ }^{\circ} \mathrm{C}$ for use. ${ }^{7,27}$

2.2.3. Synthesis of homogeneous BCHD nanocomposite scaffolds. The BCHD nanocomposite scaffolds were synthesized via the co-precipitation approach ${ }^{19}$ as described. First, CMC solution was prepared by dissolving $1 \mathrm{~g}$ of $\mathrm{CMC}$ in $50 \mathrm{~mL}$ of $\mathrm{DH}_{2} \mathrm{O}$ with continuous stirring at room temperature to obtain a perfectly transparent solution. To this solution, $1 \mathrm{~g}$ of NHA solution prepared in $50 \mathrm{~mL}$ of $\mathrm{DH}_{2} \mathrm{O}$ was added dropwise and the mixture was continuously stirred at room temperature for 4-6 hours. To this mixture, $\beta$-CD solution, made by dispersing $1 \mathrm{~g}$ of $\beta-\mathrm{CD}$ in $50 \mathrm{~mL}$ of $\mathrm{DH}_{2} \mathrm{O}$ by sonication for $30 \mathrm{~min}$, is dropped slowly to obtain a homogeneous stock solution. At this point of time, the stock solution is divided into 3 equal parts. To each part, $10 \mathrm{~mL}, 20 \mathrm{~mL}$ and $30 \mathrm{~mL}$ of DSE were added dropwise to form BCHD1, BCHD2 and, BCHD3 nanocomposite scaffolds, respectively. Then, the reaction was continued until the contents were thoroughly mixed. The reaction mixture thus obtained was kept under constant stirring at $1200 \mathrm{rpm}$ at room temperature. The $\mathrm{pH}$ of the resulting mixture was maintained at $\sim 11$ using $0.5 \mathrm{M} \mathrm{NaOH}$ to ease the nucleation of NHA followed by stirring at the same rate for another $24 \mathrm{~h}$. The slurry so obtained was aged for $48 \mathrm{~h}$ and the precipitate was harvested after repeated washing with $\mathrm{DH}_{2} \mathrm{O}$ until the filtrate became neutral. The precipitate so obtained was dried at $50{ }^{\circ} \mathrm{C}$ in a vacuum. To compare the properties of the proposed nanocomposite scaffolds, we synthesized the $\mathrm{CH}$ (CMC/nano-hydroxyapatite) nanocomposite scaffold adopting the same procedure in a $1: 1$ ratio.

\subsection{Characterization}

The morphological and chemical characterization are described in the ESI $\dagger$ under Section S.2.2.

\subsection{Statistical analysis}

Data were obtained from triplicate samples and presented as mean \pm standard deviation. A Student's $t$-test was performed for statistical analysis and the significance was defined at $p<$ $0.05(*) .{ }^{28}$

\section{Animal experiments}

\subsection{Ethical statement}

Ethical consent was obtained for all the animal trials and conducted in conformity to the experiment guidelines permitted by the Institutional Animal Ethics Committee, Jawaharlal Nehru Medical College, and Aligarh Muslim University. ${ }^{29}$

\subsection{Cerabone}

In the present study, the commercially available grafting material from Botiss (Zossen, Germany) was employed. ${ }^{30}$

\subsection{Experimental design: surgery and implantation procedure}

A total of eighteen male adult albino rats (300-350 g) were employed for the study. Animals were randomly assigned to three groups ( $n=6$ per group). The critical size $8 \mathrm{~mm}$ defect was created in the cranial bone of all animals in each group. In group (1), the defect was kept blank and treated as the control group; in group (2), cerabone (CB) was filled in the defect; and in group (3), the defect was filled with the BCHD3 nanocomposite scaffold. The intended surgical area was prepared by shaving skin and disinfection with $7.5 \%$ povidone-iodine solution (Win-Medicare, India). The surgeries were performed following the experimental procedure described in our previous studies, ${ }^{19,29}$ which involved general anaesthesia, skin incision, periosteum incision, creation of a critical defect in the parietal bone, implantation and closure of the defect that took around 30-45 minutes. The operative procedure was uneventful and postoperatively, the animals also received antibiotic cover (gentamycin) for 5 days. The rats were allowed to move freely in their cages without external support. The wounds healed normally and no abnormal behavior was noticed during the 8 week duration of the study. The animals were sacrificed after the completion of the experimental period and calvarial samples were harvested, cleaned and subjected to radiovisiographic analysis. Subsequently, these samples were decalcified for histopathological observations.

\subsection{Radiographic analyses}

For radiographic samples, the imaging system (Satelac-Acteon, $\mathrm{X}$-Mind, Italy; X-ray tube-Toshiba, Japan) was used. The radivisiographs were recorded using a radiographic sensor (Suni Plus-0543, USA). Image Analysis toolbox (MATLAB 6.1, The MathWorks Inc., Natick, MA, 2000) was used to quantify the gain in bone density (GBD) as reported previously. ${ }^{19,29}$ The results were expressed as mean \pm standard deviation (SD). The in vivo study analysis was performed using TukeyKramer multiple comparison. All results were considered to be significant at the $5 \%$ critical level. ${ }^{19}$

\subsection{Preparation of calvarial samples}

The harvested tissue of calvarial samples was fixed in formalin $(10 \%)$ for $48 \mathrm{~h}$ and kept for decalcificaton [in ethylene diamine tetra acetic acid (EDTA), 10\%] after the RVG analyses. The decalcified tissue samples were processed for paraffin sections. The sections were stained with hematoxylin-eosin (HE) and Sirius Red (SR) stains and observations were made using a light microscope (Trinocular Olympus RX40, Japan) as described in our previous studies. ${ }^{29}$

\section{Results and discussion}

\subsection{Fourier transform infrared (FTIR)}

For the elucidation of the chemical interactions between the various components present in the nanocomposite samples, FTIR analyses were carried out [Fig. 1(i)]. The FTIR scan of the carboxymethyl chitosan/nano-hydroxyapatite nanocomposite 

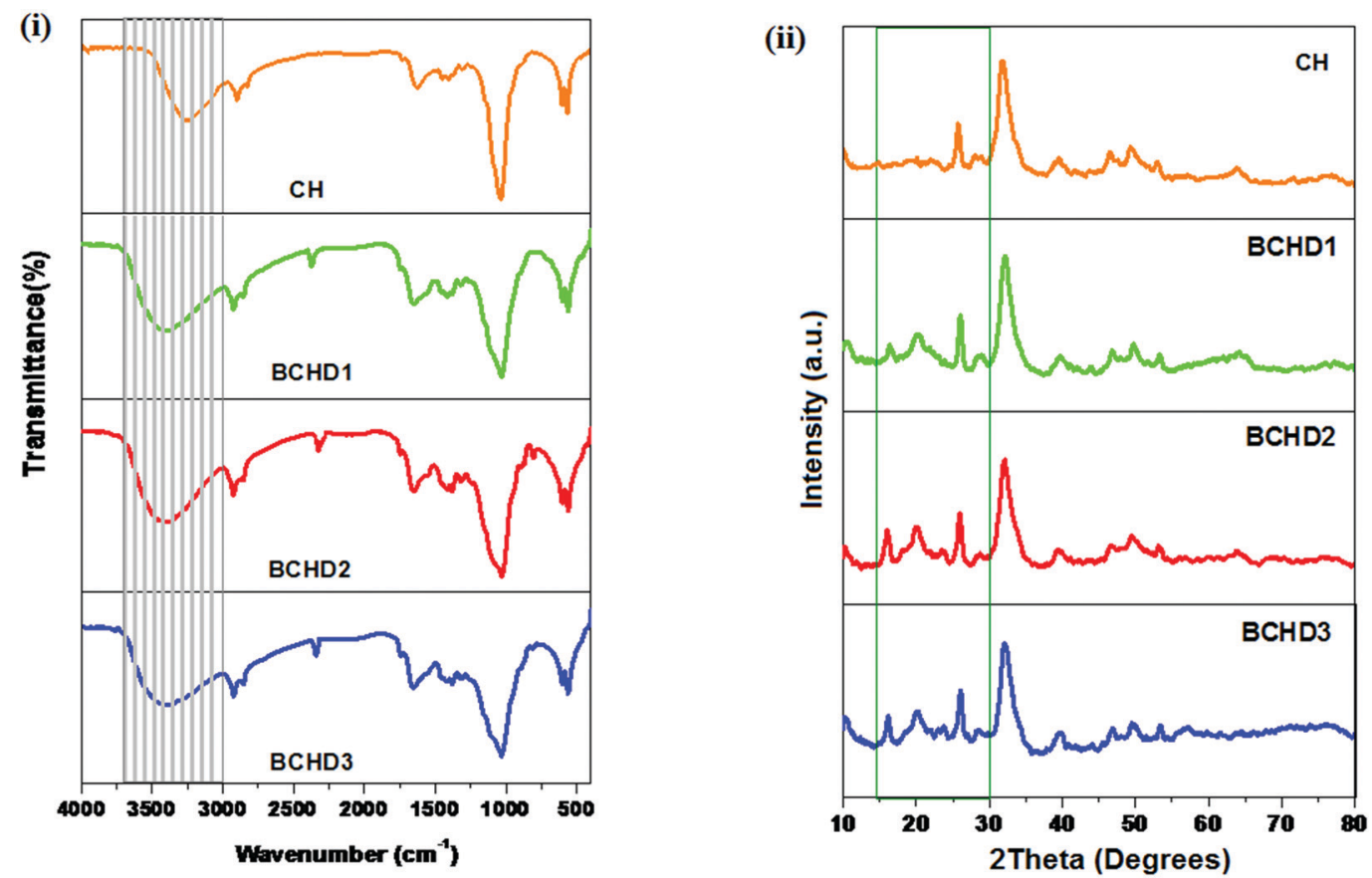

(iii)

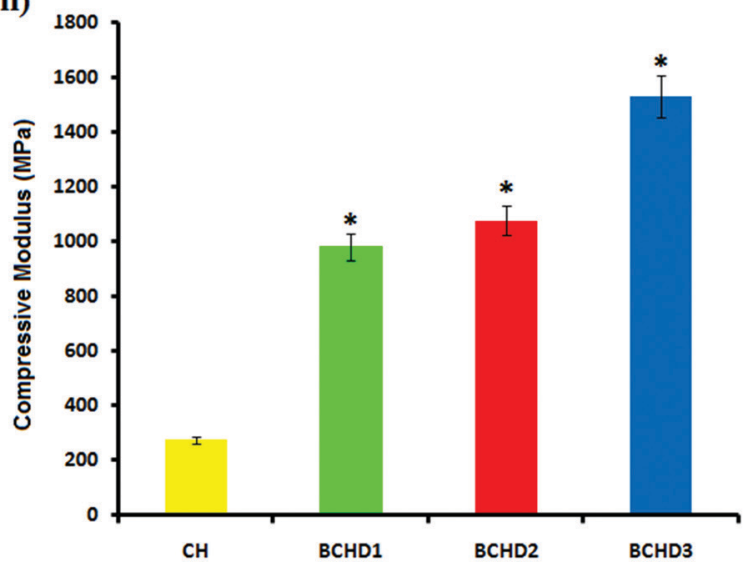

(iv)

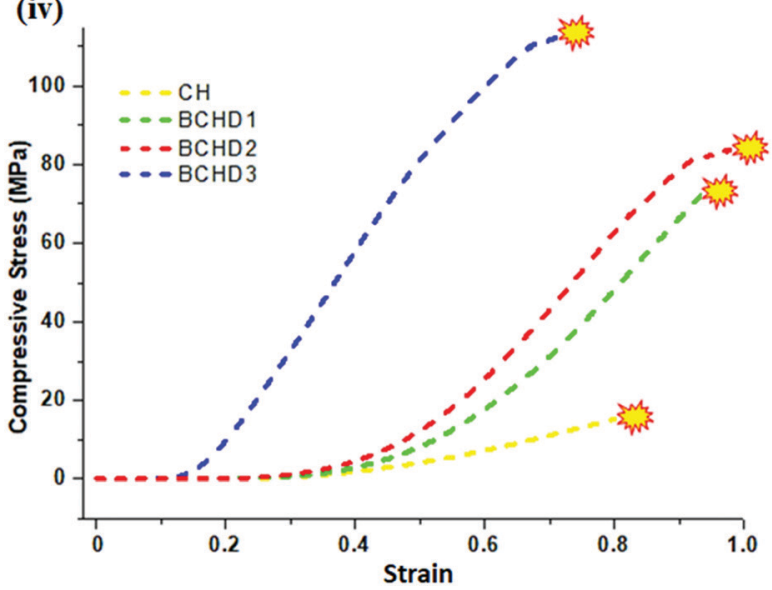

Fig. 1 FTIR spectra (i), XRD diffractograms (ii), mechanical strength analysis: compressive modulus (iii), and compressive stress-strain (iv) curves of BCHD1, BCHD2, BCHD3 and CH nanocomposite scaffolds where the fracture point has been shown by yellow color, ${ }^{\star} p<0.05$ showing the statistical difference compared with the $\mathrm{CH}$ nanocomposite scaffold.

scaffold $(\mathrm{CH})$ showed the presence of the representative bands of NHA in the range of 536-566 and 603-608 $\mathrm{cm}^{-1}$, respectively, ${ }^{31}$ corresponding to phosphate bending modes of vibrations of NHA. Also, the band characteristics of the symmetric and asymmetric deformations of $\mathrm{COO}^{-}$signifying the presence of carboxymethyl groups in CMC are shifted to 1634 and $1400 \mathrm{~cm}^{-1}$, respectively, indicating the coordinative interaction between calcium ions and CMC. ${ }^{32}$ The stretching mode of vibration of the phosphate group in NHA overlapped with the -C-O- band of CMC, resulting in a sharp peak ${ }^{33}$ at $1030 \mathrm{~cm}^{-1}$. The FTIR spectra of BCHD1, BCHD2 and BCHD3 nanocomposite scaffolds exhibited the presence of characteristic peaks of CMC and NHA; however, the stretching mode of vibration of the phosphate group in NHA overlapped with the -C-O- band of $\beta-\mathrm{CD},-\mathrm{C}-\mathrm{O}-$ band of $\mathrm{CMC}^{34}$ and $\mathrm{C}-\mathrm{O}$ stretching vibration of cellulose and hemi- cellulose present in DSE, ${ }^{15}$ resulting in a broad band ${ }^{15,19,35}$ in the region around $1020-1042 \mathrm{~cm}^{-1}$. The band representing the more hydrophilic character of $\mathrm{CMC}$ is overlapped with the $\mathrm{OH}$ stretching band of NHA, resulting in the appearance of a broad band centered at $3268 \mathrm{~cm}^{-1}$ in the $\mathrm{CH}$ nanocomposite scaffold; however the band became broader and shifted slightly to higher wavenumber and appeared in the region of $3402-3416 \mathrm{~cm}^{-1}$ with the addition of $\beta-\mathrm{CD}$ and DSE in comparison to $\mathrm{CH}$. This is possibly due to the overlapping of the $\mathrm{OH}$ stretching band of NHA with the $\mathrm{OH}$ stretching bands of CMC, $\beta$-CD and DSE, signifying the possibility of intermolecular hydrogen bonding among the components. ${ }^{15,35}$ The peak observed at $2942 \mathrm{~cm}^{-1}$ was due to the asymmetric $\mathrm{C}-\mathrm{H}$ stretching vibration from $\mathrm{CH}_{2}$ groups in $\mathrm{CMC}$ present in all the nanocomposite scaffolds. ${ }^{36-38}$ The appearance of new bands in the range of $2318-2352 \mathrm{~cm}^{-1}$ in BCHD1, BCHD2, 
and BCHD3 nanocomposite scaffolds may be attributed to the coordinative interactions between $\mathrm{Ca}^{2+}$ of $\mathrm{NHA}$ and $\mathrm{OH}$ of $\beta-\mathrm{CD}^{15}$ that is responsible to support the nucleation of apatite crystals. Thus, the analysis of the FTIR spectra confirmed that all the components used during synthesis, i.e. NHA, CMC, $\beta-\mathrm{CD}$ and DSE, have been incorporated in the final system BCHD and exhibited the expected interactions among different components present in the nanocomposite scaffolds.

\subsection{X-Ray diffraction (XRD) analysis}

The XRD patterns of $\mathrm{CH}, \mathrm{BCHD} 1, \mathrm{BCHD} 2$, and BCHD3 nanocomposite scaffolds are shown in Fig. 1(ii). The XRD spectrum of $\mathrm{CH}$ exhibited the characteristic peaks of NHA at $2 \theta=25.5$, 31.9 , etc. , in accordance with the standard diffraction pattern of NHA (PDF\# 09-0432), ${ }^{37}$ while the presence of CMC was evident by the appearance of its XRD diffraction peaks ${ }^{38}$ at $2 \theta=28.9^{\circ}$ and $46.4^{\circ}$. In the XRD spectra of BCHD1, BCHD2 and BCHD3 nanocomposite scaffolds, the characteristic peaks of NHA and CMC were observed. In addition, the presence of DSE was evident by the appearance of the peak at $2 \theta=16.2^{\circ}$ ascribed to its native cellulose and hemicellulosic components, while the other peak was overlapped with the CMC and $\beta-C D$ peaks ${ }^{19,39-41}$ and appeared at $2 \theta=20.4^{\circ}$. The appearance of a crystalline peak ${ }^{40}$ at $2 \theta=15.9^{\circ}$ also marked the presence of $\beta$-CD in BCHD1, BCHD2 and BCHD3 nanocomposite scaffolds. The broad peaks of the BCHD nanocomposite scaffolds represented low crystallinity of biological apatite, thus mimicking natural bone. ${ }^{42}$

\subsection{Mechanical strength}

In the present study, the compressive strength of the $\mathrm{CH}$, BCHD1, BCHD2 and BCHD3 nanocomposite scaffolds was tested following the guidelines for the preparation of the samples [ASTM F 451-95]. ${ }^{43}$ The results of the average compressive modulus [Fig. 1(iii)] revealed that BCHD3 exhibited a compressive modulus $[1533 \pm 2.69 \mathrm{MPa}]$ which falls in the range observed for human cortical bone and significantly higher $(p<0.05)$ than $\mathrm{CH}$, suggesting that the addition of $\beta$-CD together with DSE which is a rich source of fibers ${ }^{44}$ in the $\mathrm{CH}$ matrix has noticeably improved the mechanical parameters. The compressive stress-strain curves of the $\mathrm{CH}$, BCHD1, BCHD2 and BCHD3 nanocomposite scaffolds are shown in Fig. 1(iv), where it can be observed that in the initial phase of compression, there was a sharp increase in stress with subsequent decrease until the point of fracture was attained. The increased intermolecular hydrogen bonding among nano-hydroxyapatite, cellulose and hemicellulose present in DSE, CMC and $\beta$-CD moieties may possibly be the reason for enhanced mechanical parameters displayed by the BCHD nanocomposite scaffolds, which might create a rigid framework with suitable mechanical properties, thus facilitating their clinical applications. ${ }^{40}$

\subsection{Transmission electron microscopy (TEM) and contact angle measurements}

The morphologies of BCHD1, BCHD2, BCHD3 and $\mathrm{CH}$ nanocomposite scaffolds were characterized by TEM. Based on the results as displayed in Fig. 2(A-D), needle-like particles were homogeneously distributed in the case of BCHD1, BCHD2 and BCHD3 having an average particle size in the range of 18-25 nm which was found to decrease with an increase in the concentration of DSE in BCHD3 as shown in Fig. 2C. In the case of $\mathrm{CH}$, the average particle size varied in the range of $30-35 \mathrm{~nm}$ and the particles were observed to be comparatively crowded resulting in the formation of the aggregated structure. ${ }^{32,45}$ The consistent dispersion of particles in the case of the proposed nanocomposite scaffolds may be due to the interactions of DSE with CMC, NHA and $\beta$-CD. As the size of the particles was found to be a function of the concentration of DSE, hence, the DSE appeared to play a key
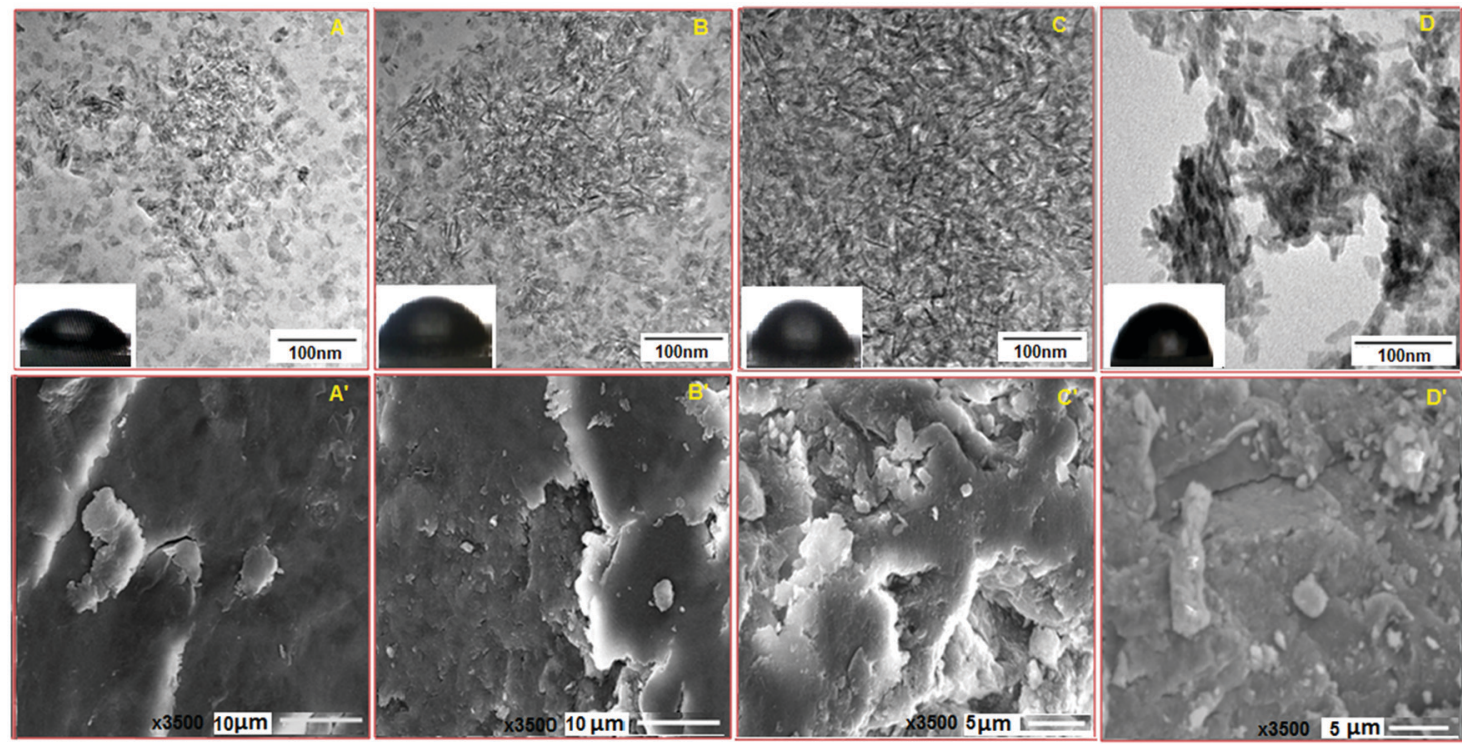

Fig. 2 Representative TEM micrographs of (A) BCHD1, (B) BCHD2, (C) BCHD3, and (D) CH nanocomposite scaffolds [scale bar: $100 \mathrm{~nm}$ ] with the inset showing water contact angles and their respective SEM images $\left[A^{\prime}-D^{\prime}\right.$ ] [scale bar: $10 \mu \mathrm{m}$, magnification: $\times 3500$ ]. 

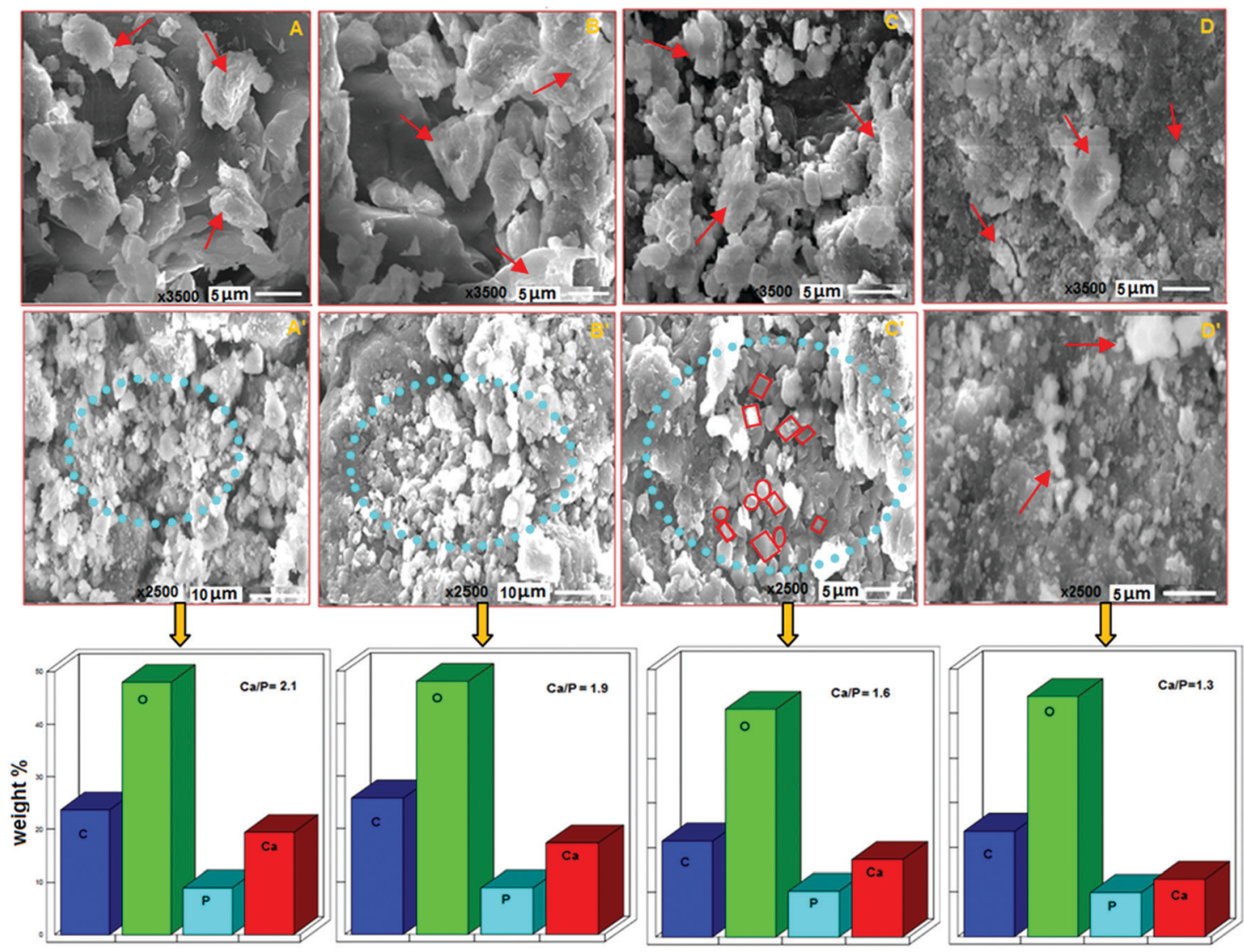

Fig. 3 Characteristic SEM micrographs illustrating the bioactivity profile by the SBF study of $[A] B C H D 1,[B] B C H D 2,[C] B C H D 3, a n d[D] C H$ nanocomposite scaffolds at 4 weeks [magnification: $\times 3500$, scale bar: $5 \mu \mathrm{m}$ ] and at 8 weeks [A'-D'] [magnification: $\times 2500$, scale bar: $10 \mu \mathrm{m}$, respectively, with their respective EDX micrographs recorded after 8 weeks.

role in controlling the size of the particles. ${ }^{46,47}$ These results were found to be in line with the previous literature indicating the role of DSE as a size and shape modifying agent. ${ }^{35}$ The water contact angle studies conducted on BCHD1, BCHD2, BCHD3 and $\mathrm{CH}$ nanocomposite scaffolds [Fig. 2(A-D) inset] showed that the contact angle was smaller in the case of BCHD nanocomposite scaffolds with a WCA of $32.6^{\circ}$ for BCHD1 and $38.9^{\circ}$ for BCHD2, while BCHD3 showed a moderately hydrophilic surface with a WCA of $42.5^{\circ}$ and was thus found to be most suitable for the adhesion and proliferation of the cells in comparison to relatively hydrophobic $\mathrm{CH}$ with a contact angle of $69.4^{\circ}$ in view of the synergic addition of the high degree of $-\mathrm{OH}$ groups present in NHA, CMC, $\beta-\mathrm{CD}$ and DSE. $^{48}$

\subsection{SEM}

SEM analysis was conducted in order to investigate the microstructure and surface morphologies of BCHD1, BCHD2, BCHD3 and $\mathrm{CH}$ nanocomposite scaffolds. Fig. $2\left(\mathrm{~A}^{\prime}-\mathrm{D}^{\prime}\right)$ represents the porous and rougher surface morphologies of BCHD1, BCHD2,

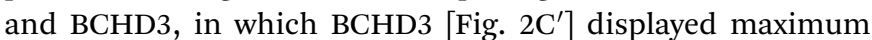
roughness compared with the $\mathrm{CH}$ nanocomposite scaffold where CMC imparted smoothness to the surface [Fig. 2D']. ${ }^{38,49}$ However, the introduction of DSE and $\beta$-CD significantly enhanced the roughness in the nanocomposite scaffolds ${ }^{15,19}$ owing to the presence of intermolecular $\mathrm{H}$-bonding. Therefore, the synergism among NHA, CMC, $\beta-\mathrm{CD}$ and DSE in BCHD nanocomposite scaffolds caused an increased surface area and an interconnected structure with a sufficiently higher porosity appropriate for the transportation of extracellular fluids, nutrients, and hormones to cells ${ }^{50}$ and thus making it a suitable platform for applications in BTE.

\subsection{In vitro calcification assay and EDS}

The representative SEM images at 4 weeks of in vitro calcification conducted on BCHD1, BCHD2, BCHD3 and $\mathrm{CH}$ nanocomposite scaffolds are shown in Fig. 3(A-D). Having plenty of carboxyl and OH groups, BCHD nanocomposite scaffolds showed flattened and fused flaky apatite growth (shown by red arrows), resulting from the combination of calcium ions with more number of carboxyl and hydroxyl groups present on their surfaces in comparison to the $\mathrm{CH}$ nanocomposite scaffold. In accordance with earlier studies, ${ }^{51,52}$ this observation indicated the catalytic effect of the hydroxyl and carboxyl groups in inducing apatite formation. At 8 weeks [Fig. 3 $\left(\mathrm{A}^{\prime}-\mathrm{D}^{\prime}\right)$ ], though the carboxyl groups of $\mathrm{CMC}$ present in $\mathrm{CH}$ acted as the template for subsequent biomimetic deposition, ${ }^{43,53}$ the mineralization got highly increased in all BCHD nanocomposite scaffolds (shown in circular regions) relative to $\mathrm{CH}$. These observations may be 

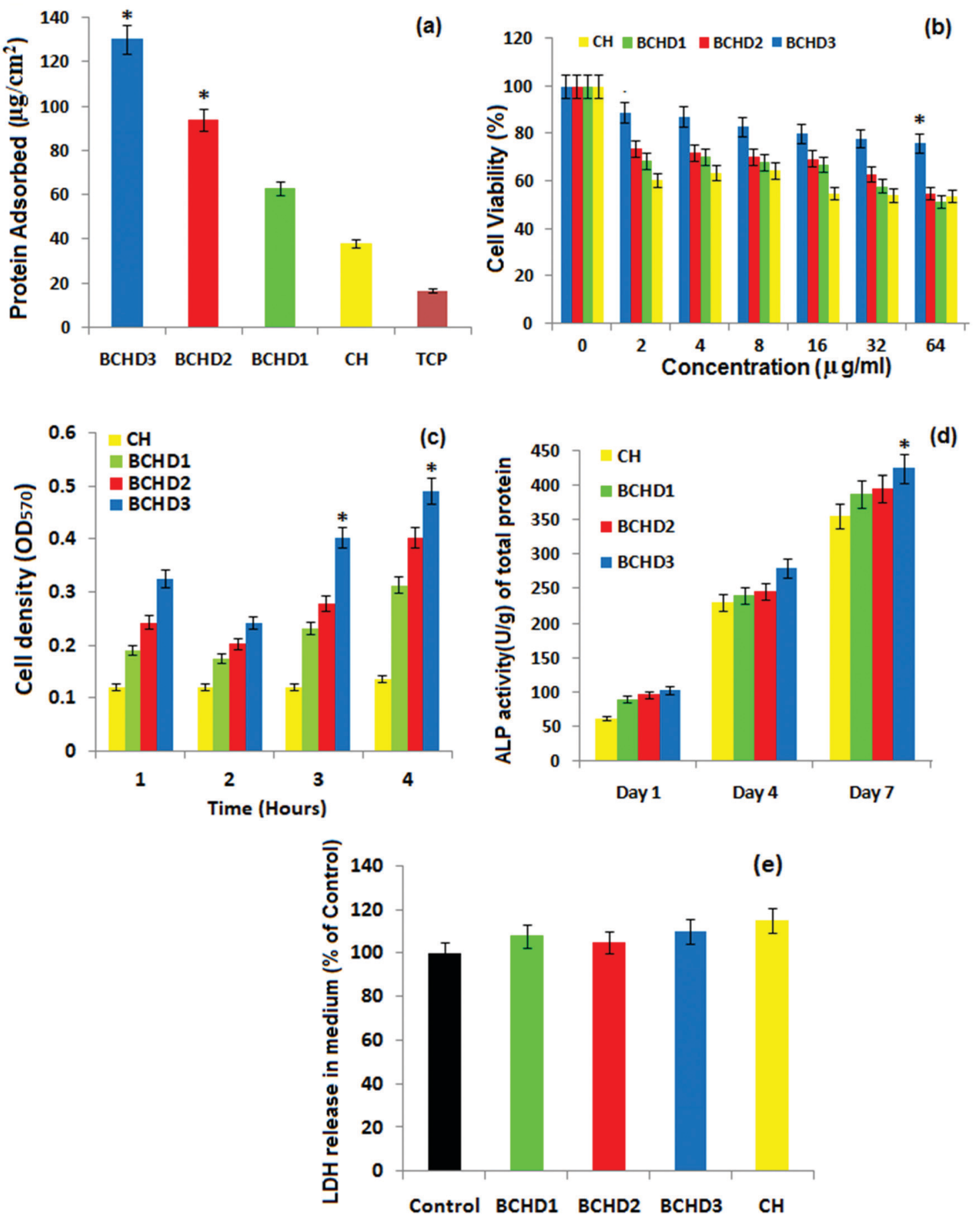

Fig. 4 Biocompatibility studies: (a) protein adsorption assay, (b) cell viability assay, (c) MG-63 cell density measured at 1-4 hours, (d) alkaline phosphatase activity, (e) LDH assays of BCHD1, BCHD2, BCHD3, and $\mathrm{CH}$ nanocomposite scaffolds. ${ }^{*} p<0.05$ shows a statistical difference compared to the $\mathrm{CH}$ nanocomposite scaffold.

understood in terms of the relatively rougher surface exhibited by the BCHD nanocomposite scaffolds, leading to enhanced osteoconductivity and bioactivity. Also, it was noticed that the apatite formation occurred as irregular granular depositions comprising mainly spherical and rectangular granules in the case of BCHD2 which appeared to get denser in the case of BCHD3, as shown in Fig. $3 \mathrm{C}^{\prime}$, inferring that with an increase in the concentration of DSE in BCHD3, more apatite nucleation occurred by the consumption of calcium and phosphate ion precursors present in the surrounding fluid. ${ }^{54}$ These results possibly indicate the ability of $\beta$-CD and DSE as a mineralization inducer where DSE is a rich source of minerals essential for bone formation. ${ }^{44}$ These results were found to be in agreement with the former results. ${ }^{15,19}$ 
The EDS results of the SBF treated BCHD1, BCHD2, BCHD3 and $\mathrm{CH}$ nanocomposite scaffolds after 8 weeks incubation [Fig. 3] clearly showed that the composition and phase resembled the mineral component of the bone. The apatite formed in the case of BCHD nanocomposite scaffolds exhibited $^{55}$ a semiquantitative ratio of $\mathrm{Ca} / \mathrm{P}$ in the close proximity of 1.6-2.1 which is the characteristic range found in natural bone $^{41}$ in comparison to the ratio (1.3) obtained in the case of the $\mathrm{CH}$ nanocomposite scaffold.

These results provided a fundamental desirable characteristic of a bioactive material that facilitates osteointegration in vivo.

\subsection{Protein adsorption assay}

Protein adsorption is a crucial index to investigate the biocompatible nature of a scaffold for bone engineering applications. Nanocomposite scaffolds with varying amount of DSE were incubated in FBS solution and compared with $\mathrm{CH}$ to investigate their protein adsorption ability. The adsorption of protein on the surface of $\mathrm{CH}, \mathrm{BCHD} 1, \mathrm{BCHD} 2$, and BCHD3 nanocomposite scaffolds and a tissue culture plate (TCP) is shown in Fig. 4a. It was observed that all the BCHD nanocomposite scaffolds exhibited significantly higher protein adsorption compared with $\mathrm{CH}$ with a maximum average value of $130 \pm 41 \mu \mathrm{g} \mathrm{cm}^{-2}$ shown by BCHD3. The addition of $\beta$-CD and date seed extracts to the $\mathrm{CH}$ matrix enhanced the surface roughness, thus increasing protein-binding channels. These observations suggested the favorable bioaffinity of the BCHD3 nanocomposite scaffold to a range of proteins present in the extracellular matrix such as fibronectin, glycoprotein and the different growth factors that could assist adhesion, proliferation, and differentiation of cells both in vitro and in vivo. ${ }^{56}$

\subsection{Cell proliferation: MTT assay}

The cell proliferation was quantitatively evaluated by MTT assay by culturing human osteoblast like MG-63 cells on $\mathrm{CH}$, BCHD1, BCHD2 and BCHD3 nanocomposite scaffolds at various concentrations (0-64 $\left.\mu \mathrm{g} \mathrm{mL} \mathrm{m}^{-1}\right)$, as shown in Fig. 4b. The BCHD1, BCHD2 and BCHD3 nanocomposite scaffolds inferred higher metabolic activity of the cells compared to $\mathrm{CH}$; however, BCHD3 showed significant cell viability even at higher concentration validating its proficiency in increasing biocompatibility. The optical density value was also detected at a series of time points of 1-4 hours to evaluate cell proliferation (Fig. 4c). Clearly, the results showed higher cell proliferation in the case of the BCHD nanocomposite scaffolds compared with the $\mathrm{CH}$ nanocomposite scaffold with BCHD3 displaying significantly higher $(p<0.05)$ cell density at 3 and 4 hours compared with $\mathrm{CH}$. The synergic combination of CMC ${ }^{36} \mathrm{NHA}, \beta-\mathrm{CD}$ and DSE may possibly lead to a well-interconnected rougher and porous surface in the case of BCHD nanocomposite scaffolds, which is responsible for better structural stability that plays a crucial function in cell attachment and proliferation. ${ }^{57}$

\subsection{Alkaline phosphatase (ALP) assay}

Generally, ALP assists in the calcium deposition in addition to initial bone mineralization. Therefore, as part of our in vitro investigation, the ALP activity of MG63 cells subjected to BCHD1, BCHD2, BCHD3 and $\mathrm{CH}$ samples was monitored and is shown in Fig. 4c. The results illustrated that the BCHD1, BCHD2 and BCHD3 nanocomposite scaffolds significantly increased the expression of ALP $(p<0.05)$ in comparison to the $\mathrm{CH}$ at all the tested time points of 1,4 and 7 days. The superior activity might be attributed to the presence of $\beta$-CD and DSE in the $\mathrm{CH}$ matrix, which impart porosity and larger surface area concentrating more proteins and growth factors from the culture, hence providing an effective substrate for cellular differentiation stimulating the process of osteogenesis. ${ }^{15,19}$

\subsection{LDH assay}

To further understand the biocompatible characteristics, LDH leakage assays of the BCHD1, BCHD2, BCHD3 and $\mathrm{CH}$ nanocomposite scaffolds were conducted and the results are shown in Fig. $4 \mathrm{~d}$ after $24 \mathrm{~h}$ of co-culture with MG63 cells. The extent of damage to the cell membrane is indicated by evaluating the percentage of $\mathrm{LDH}$ leakage since $\mathrm{LDH}$ is a stable cytoplasmic enzyme, which gets released if the plasma membrane integrity is compromised. ${ }^{56}$ It is speculated that in comparison to the control, no significant increase $(p>0.05)$ was observed in the $\mathrm{LDH}$ releasing level, further confirming the cytocompatability of the nanocomposite scaffolds. However the LDH level was found to be slightly lower in the case of quaternary systems relative to $\mathrm{CH}$, warranting the enhanced biocompatibility of the proposed nanocomposite scaffolds ${ }^{58}$ with the addition of $\beta$-cyclodextrin and date seed extracts to the $\mathrm{CH}$ matrix, resulting in enhanced intermolecular interactions. ${ }^{15,19}$ These observations run parallel to the previous reports and further confirmed the biocompatibility of the BCHD nanocomposite scaffolds.

\section{In vivo study analysis}

\subsection{Clinical observations}

The visual inspection immediately after the creation of calvarial defects affirmed that the dura mater remains intact in all the groups and there were no complications emerged from the craniotomy. From the clinical aspect, all rats healed well and were observed to gain weight throughout the study period.

\subsection{Radiographic and histological assessment}

Following in vitro characterizations, preliminary in vivo investigation was carried out. The histological analysis of hematoxylin and eosin (H\&E) and Sirius red (SR) stained sections in the control (blank) group after 4 weeks [Fig. 5 control panel] showed no sign of new bone formation at the defect site. However, active osteoblasts were seen lined up at the margin of the defect and the defect itself was occupied with the fibrous tissue primarily made up of the collagen fibre. ${ }^{19,29}$ The control group at 8 weeks also did not reveal the bone formation at the site of the defect; however, the fibrous tissue which was filling the defect showed increased vascularisation by invading newly formed blood vessels [Fig. 6 control panel]. The RVG results of GBD (gain in bone density) for the control 


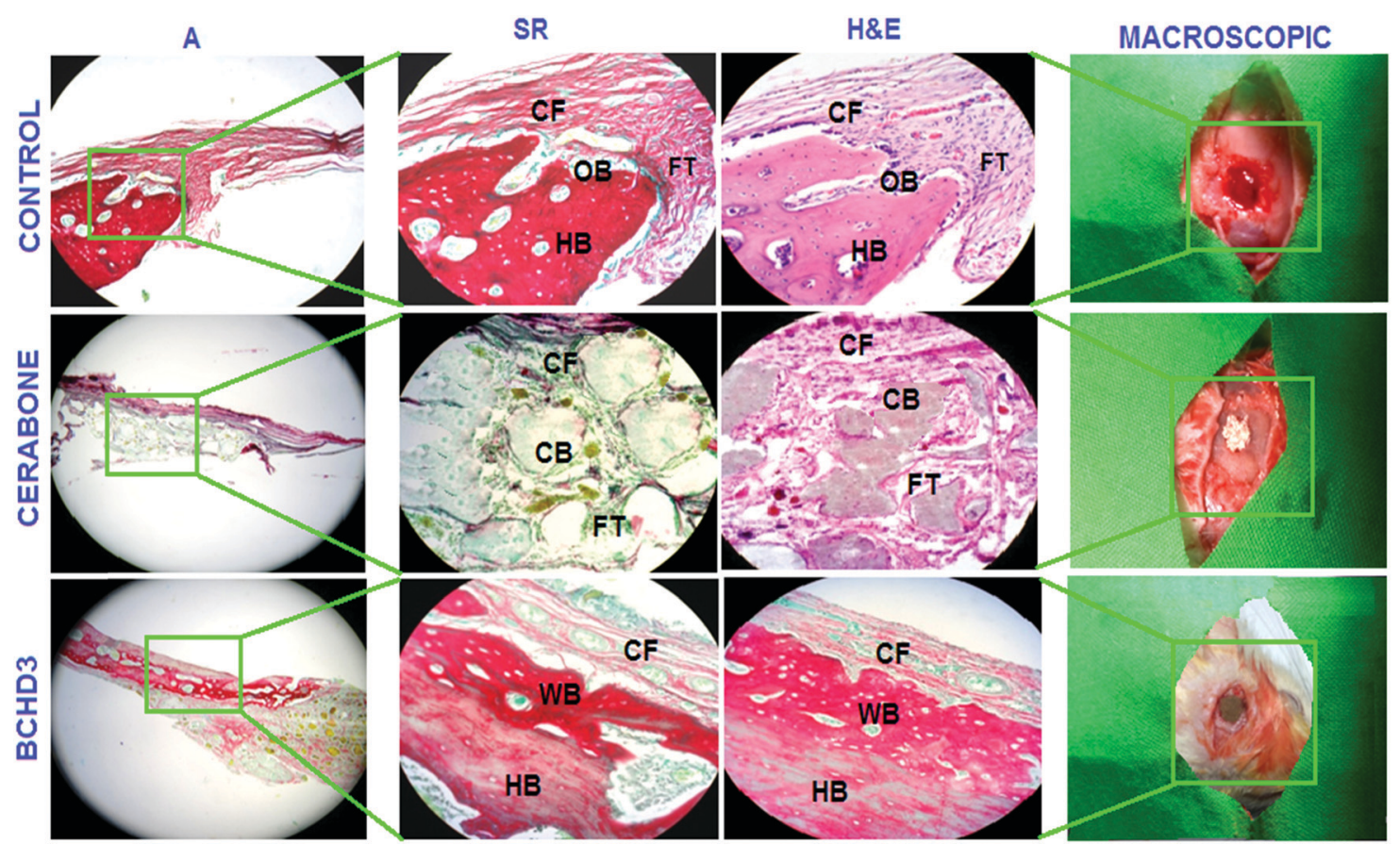

Fig. 5 Histopathology (H\&E and SR stains) after 4 weeks post-implantation of the control group, cerabone group and BCHD3 group and their macroscopic view; initial magnification: $\times 100$ (shown by A) and $\times 200$. [OB: osteoblasts, CF: collagen fibre, CB: cerabone, HB: host bone, FT: fibrous tissue, and WB: woven bone].

group at 4 and 8 weeks were found to be $26.60 \pm 0.18 \%$ and $37.40 \pm 1.54 \%$, respectively [Fig. 7 (i, ii) control panels], representing negligible gain which was also in conformity with the histological findings of these groups. The cerabone group at 4 weeks [Fig. 5 cerabone panel] revealed the absence of new bone formation and the defect was seen to be filled with collagen fibre and cerabone with a GBD of $37.71 \pm 1.12 \%$ [Fig. 7(i)]. At 8 weeks, the cerabone group showed a mixed picture having fibrous tissue, some bone and clumps of cerabone as well, thereby suggesting the partial utilization of cerabone for bone formation $^{29}$ [Fig. 6 cerabone panel] and the GBD was found to be $86.0 \pm 1.4 \%$ [Fig. 7 (ii)] which supported

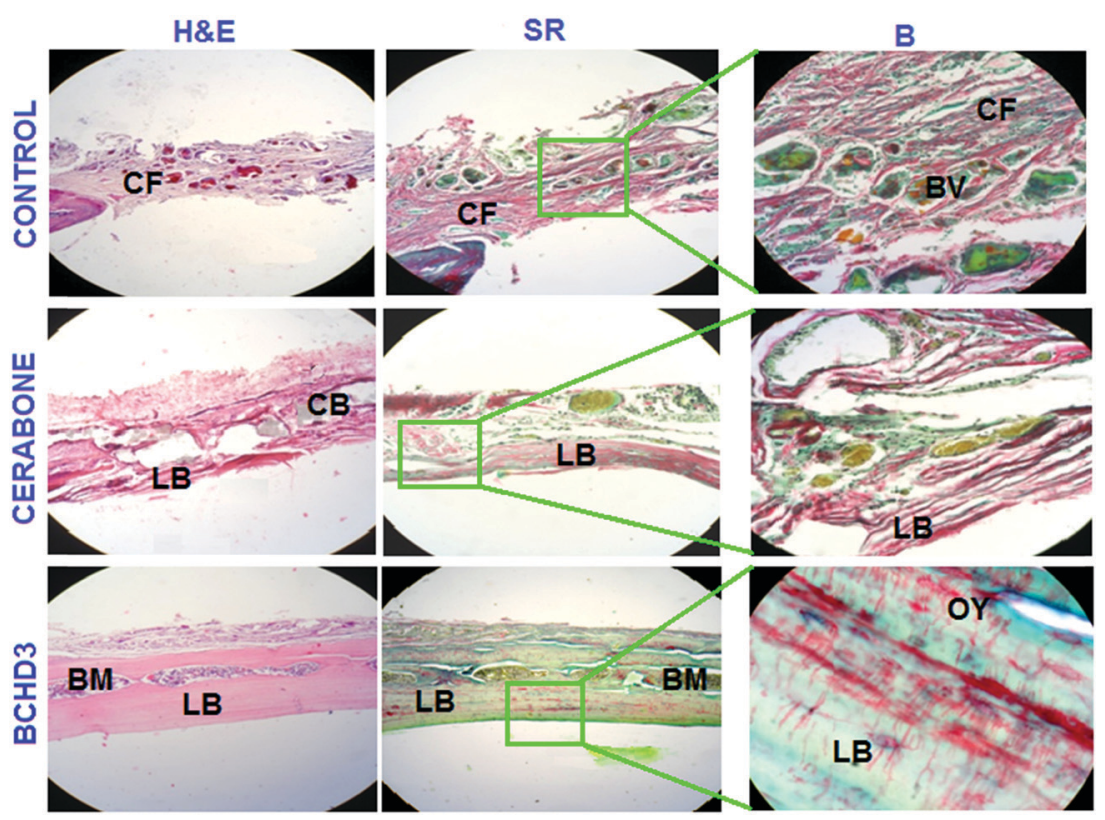

Fig. 6 Histopathology (H\&E and SR stains) after 8 weeks post-implantation in the control, cerabone, and BCHD3 experimental groups. Initial magnification: $\times 100$, and B represents maginified view at $\times 200$. [CF: collagen fibre, BV: blood vessels, CB: cerabone, LB: lamellar bone, BM: bone marrow, and OY: osteocytes with multiple processess]. 

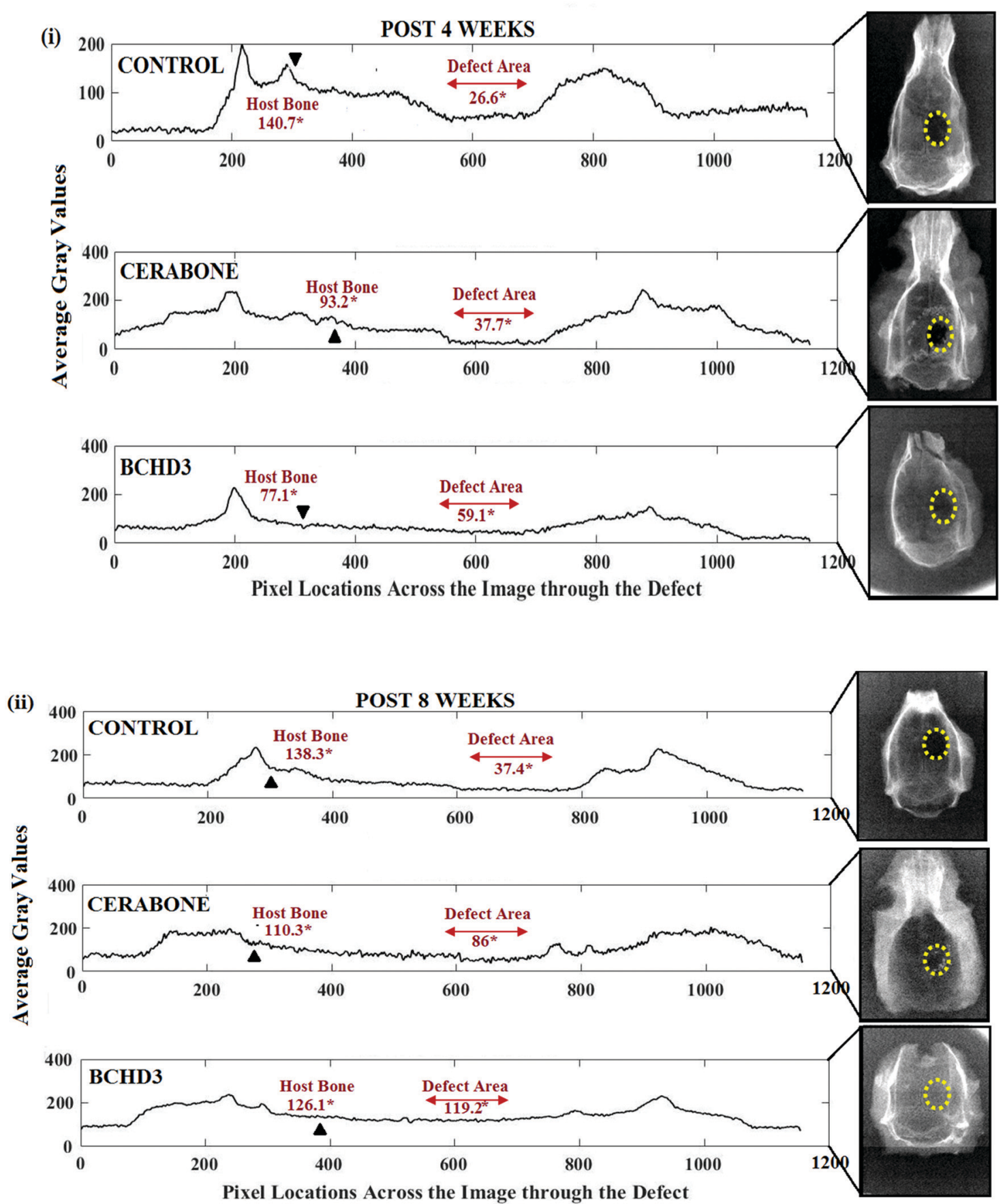

Fig. 7 Gray value profiles of the control, cerabone, and BCHD3 experimental groups after the time period of 4 and 8 weeks. Asterisk indicates the average gray values in the host bone region (indicated by $\mathbf{\Lambda}$ ) and defect regions.

the histological findings of partial bone formation. In the case of the BCHD3 group, at 4 weeks, the RVG analysis revealed a GBD of $59 \% \pm 1.13 \%$, [Fig. 7(i)]; thus, it is really startling and gratifying to note that the BCHD3 group is superior in promoting the bone formation to the extent that even at 4 weeks, there is distinct evidence of woven bone formation. Moreover, by 8 weeks, when there is no bone formation in the control and moderate bone formation in $\mathrm{CB}$, the BCHD3 group showed not only newly formed bone but also remodelled lamellar bone with a GBD of
$94.5 \pm 1.81$ [Fig. 7(ii)]. Notably, in the case of the BCHD3 group at 8 weeks, there was the appearance of bone marrow that is rich in mesenchymal stem cells and osteoblasts and thus marks the critical aspect for the regeneration of bone tissue. From these observations, it may be concluded that BCHD3 not only promoted osteoregeneration, osteoinduction, osteointegration and advanced calcification, but also successfully bridged the critical size defect within 8 weeks even without the use of any growth stimulators and prior cell seeding. 
The possible reason for the exceptional performance of BCHD3 may be accounted in terms of synergism among its various constituents. NHA has an ion-inducing effect and releases $\mathrm{PO}_{4}{ }^{3-}$ and $\mathrm{Ca}^{2+}$ in the physiological environment, which constitutes a key duo for proper bone mineral acquisition and maintenance, thus participating in the process of osteogenic differentiation leading to new bone formation. ${ }^{36}$ Next, the component $\beta$-CD, being biologically inert, has been approved by the US FDA as the pharmaceutical excipient for numerous drug formulations. Recently, it was reported to show a robust bone anabolic effect in vivo. ${ }^{59}$ Another component CMC, being a derivative of carboxymethyl chitin, has also been found to be efficient in causing nucleation of apatite and osteogenesis due to the presence of carboxyl groups. ${ }^{60}$ This causes the induction of inflammatory cytokines and growth factors and thus accounted for restoring cartilage. ${ }^{43}$ On the other hand, besides being a rich source of proteins, fibres, antioxidants and minerals, DSE has been reported to include several important chemical constituents such as alkaloids, carbohydrates, tannins, etc., which mainly comprise nitrogen and oxygen heteroatoms, that play a crucial role in activating the process of bone formation as reported previously. ${ }^{40,61}$ Furthermore, plant extracts have also been documented to increase the antioxidant defenses and decrease the tissue inflammation, along with accelerating tissue vascularisation and proliferation of the bone cells. They were also reported to stimulate bone repair, promote osteogenesis, speed up calcification, and endorse bone callus formation and mineralization. ${ }^{62}$ Hence, the higher concentration of DSE in the BCHD3 nanocomposite scaffold exhibited a combinatorial effect with other constituents, leading to improvement in the osteoinductivity by enhancing roughness, surface area and topography, therefore influencing the differentiation of stem cells and accelerating the process of new bone formation on the fractured region. ${ }^{63-65}$ Subsequently, these results will thus serve as a cornerstone for future research focused on the design of bioinspired conductive nanocomposite scaffolds incorporating plant extracts for bone tissue regeneration.

\section{Conclusion}

The present work was intended to invest an agricultural waste product into the pharmaceutical domain, which can offer therapeutic value with a socioeconomic cost. Valorization of these natural origin materials could condense the utilization of synthetic materials for scaffold fabrication, thus avoiding the release of harmful byproducts during fabrication. ${ }^{40}$ The BCHD nanocomposite scaffolds were efficaciously developed by the co-precipitation approach for bone regeneration applications. To summarize, the nanocomposite scaffolds showed a porous and interconnected structure essential for the bone regeneration process. Among various concentrations taken into account, BCHD3, with the maximum DSE content, has stood out as a promising alternative showing excellent bioactivity and cytocompatibility with MG-63 cell lines. Also, the BCHD nanocomposite scaffolds were found to be successful in advancing cell adhesion and ALP activity besides causing calcium ion deposition, which are regarded as the key parameters for active bone formation. These results demonstrated the portentous role of natural-based quaternary nanocomposite scaffolds with minimum side effects and reduced costs, and they may prove efficacious in treating bone defects.

\section{Conflicts of interest}

There are no conflicts of interest to declare.

\section{Acknowledgements}

Dr Reshma Jolly is thankful to the Council of Scientific and Industrial Research [CSIR, file no. 09/112 (575)18-EMR-I] for financial assistance. The authors acknowledge grant supports from the DRS-II, FIST and PURSE to the Department of Chemistry, A. M. U., Aligarh. Dr Reshma Jolly is grateful to Dr Muhamed Faizal and Dr Bijo Elsy from the Department of Anatomy, JNMC, A. M. U., for their help in histological analysis. Dr Bhanu Pratap Singh, from the CSIR - National Physical Laboratory, is highly acknowledged for kind help in mechanical parameter characterization and analysis.

\section{References}

1 X. Sun, Y. Kang, J. Bao, Y. Zhang, Y. Yang and X. Zhou, Modeling vascularized bone regeneration within a porous biodegradable CaP scaffold loaded with growth factors, Biomaterials, 2013, 34, 4971-4981, DOI: 10.1016/ j.biomaterials.2013.03.015.

2 J. P. Kumar, N. Bhardwaj and B. B. Mandal, Cross-linked silk sericin-gelatin $2 \mathrm{D}$ and $3 \mathrm{D}$ matrices for prospective tissue engineering applications, $R S C A d v$., 2016, 6, 105125-105136, DOI: 10.1039/c6ra18654h.

3 S. Yan, P. Xia, S. Xu, K. Zhang, G. Li, L. Cui and J. Yin, Nanocomposite Porous Microcarriers Based on StrontiumSubstituted HA-g-Poly( $\gamma$-benzyl-L-glutamate) for Bone Tissue Engineering, ACS Appl. Mater. Interfaces, 2018, 10, 16270-16281, DOI: 10.1021/acsami.8b02448.

4 T. Gong, J. Xie, J. Liao, T. Zhang, S. Lin and Y. Lin, Nanomaterials and bone regeneration, Bone Res., 2015, 3, 15029, DOI: 10.1038/boneres.2015.29.

5 S. Samavedi, A. R. Whittington and A. S. Goldstein, Calcium phosphate ceramics in bone tissue engineering: A review of properties and their influence on cell behavior, Acta Biomater., 2013, 9, 8037-8045, DOI: 10.1016/j.actbio.2013.06.014.

6 M. Mehrali, E. Moghaddam, S. F. S. Shirazi, S. Baradaran, M. Mehrali, S. T. Latibari, H. S. C. Metselaar, N. A. Kadri, K. Zandi and N. A. A. Osman, Synthesis, Mechanical Properties, and in Vitro Biocompatibility with Osteoblasts of Calcium Silicate-Reduced Graphene Oxide Composites, ACS Appl. Mater. Interfaces, 2014, 6, 3947-3962, DOI: 10.1021/am500845x|.

7 M. Hasan and A. Mohieldein, In Vivo Evaluation of Anti Diabetic, Hypolipidemic, Antioxidative Activities of Saudi 
Date Seed Extract on Streptozotocin Induced Diabetic Rats, J. Clin. Diagn. Res., 2016, 10(3), FF06-FF12, DOI: 10.7860/ JCDR/2016/16879.7419.

8 J. M. Song, S. H. Shin, Y. D. Kim, J. Y. Lee, Y. J. Baek, S. Y. Yoon and H. S. Kim., Comparative study of chitosan/ fibroin-hydroxyapatite and collagen membranes for guided bone regeneration in rat calvarial defects: micro-computed tomography analysis, Int. J. Oral Sci., 2014, 6, 87-93, DOI: 10.1038/ijos.2014.16.

9 J. Nourmohammadi, A. Ghaee and S. H. Liavali, Preparation and characterization of bioactive composite scaffolds from polycaprolactone nanofibers-chitosan-oxidized starch for bone regeneration, Carbohydr. Polym., 2016, 138, 172-179, DOI: 10.1016/j.carbpol.2015.11.055.

10 Z. Shariatinia, Carboxymethyl chitosan: Properties and biomedical applications, Int. J. Biol. Macromol., 2018, 120, 1406-1419, DOI: 10.1016/j.ijbiomac.2018.09.131.

11 D. Gopi, S. Nithiya, E. Shinyjoy, D. Rajeswari and L. Kavitha, Carbon Nanotubes/Carboxymethyl Chitosan/Mineralized Hydroxyapatite Composite Coating on Ti-6Al-4V Alloy for Improved Mechanical and Biological Properties, Ind. Eng. Chem. Res., 2014, 53, 7660-7669, DOI: 10.1021/ie403903q.

12 L. Zhao, B. Zhu, Y. Jia, W. Hou and C. Su, Preparation of Biocompatible Carboxymethyl Chitosan Nanoparticles for Delivery of Antibiotic Drug, BioMed Res. Int., 2013, 1-7, DOI: $10.1155 / 2013 / 236469$.

13 R. Periasamy, A systematic review on the significant roles of cyclodextrins in the construction of supramolecular systems and their potential usage in various fields, J. Carbohydr. Chem., 2020, 39(5-6), 189-216, DOI: 10.1080/07328303.2020.1792919.

14 V. D. F. Manakker, T. Vermonden, V. C. F. Nostrum and W. E. Hennink, Cyclodextrin-based polymeric materials: synthesis, properties, and pharmaceutical/biomedical applications, Biomacromolecules, 2009, 10, 3157-3175, DOI: 10.1016/j.actbio.2018.06.033.

15 M. Shakir, R. Jolly, M. S. Khan, A. Rauf and S. Kazmi, Nanohydroxyapatite/ $\beta-\mathrm{CD} /$ chitosan nanocomposite for potential applications in bone tissue engineering, Int. J. Biol. Macromol., 2016, 93, 276-289, DOI: 10.1016/j.ijbiomac.2016.08.046.

16 W. K. Grier, A. S. Tiffany, M. D. Ramsey and B. A. C. Harley, Incorporating beta-cyclodextrin into collagen scaffolds to sequester growth factors and modulate mesenchymal stem cell activity, Acta Biomater., 2018, 76, 116-125, DOI: 10.1016/ j.actbio.2018.06.033.

17 T. G. B. Deluzio, K. I. Penev and K. Mequanint, Cyclodextrin Inclusion Complexes as Potential Oxygen Delivery Vehicles in Tissue Engineering, J. Biomater. Tissue Eng., 2014, 4, 957-966.

18 S. Majumdar, X. Wang, S. D. Sommerfeld, J. J. Chae, E.N. Athanasopoulou, L. S. Shores, X. Duan, L. M. Amzel, F. Stellacci, O. Schein, Q. Guo, A. Singh and J. H. Elisseeff, Cyclodextrin Modulated Type I Collagen Self-Assembly to Engineer Biomimetic Cornea Implants, Adv. Funct. Mater., 2018, 28, 1804076, DOI: 10.1002/adfm.201804076.

19 R. Jolly, A. A. Khan, S. S. Ahmed, S. Alam, S. Kazmi, M. Owais, M. A. Farooqi and M. Shakir, Bioactive Phoenix dactylifera seeds incorporated chitosan/hydroxyapatite nanoconjugate for prospective bone tissue engineering applications: A bio-synergistic approach, Mater. Sci. Eng., C, 2020, 109, 110554, DOI: 10.1016/j.msec.2019.110554.

20 A. A. Salama, N. M. Ismael and M. M. Megeed, Using Date Seed Powder Nanoparticles and Infusion as a Sustainable Source of Nutraceuticals, J. Food Nutr. Sci., 2019, 7(3), 39-48, DOI: $10.11648 /$ j.jfns.20190703.11.

21 T. P. Kalantaripour, M. Asadi-Shekaari, M. Basiri and N. A. Gholaamhosseinian, Cerebroprotective effect of date seed extract (Phoenix dactylifera) on focal cerebral ischemia in male rats, J. Biol. Sci., 2012, 12, 180-185, DOI: 10.3923/ jbs.2012.180.185.

22 K. Joyce, G. T. Fabra and Y. Bozkurt, et al., Bioactive potential of natural biomaterials: identification, retention and assessment of biological properties, Signal Transduction Targeted Ther., 2021, 6, 122, DOI: 10.1038/s41392-021-00512-8.

23 D. Tang, R. S. Tare, L.-Y. Yang, D. F. Williams, K.-L. Ou and R. O. C. Oreffo, Biofabrication of bone tissue: approaches, challenges and translation for bone regeneration, Biomaterials, 2016, 83, 363-382, DOI: 10.1016/j.biomaterials.2016.01.024.

24 B. Stevens, Y. Yang, A. Mohandas, B. Stucker and K. T. Nguyen, A Review of Materials, Fabrication Methods, and Strategies Used to Enhance Bone Regeneration in Engineered Bone Tissues, J. Biomed. Mater. Res., Part B, 2007, 85(2), 573-582, DOI: 10.1002/jbm.b.30962.

25 M. Shakir, R. Jolly, M. S. Khan, N. Iram, T. K. Sharma and S. I. Al-Resayes, Synthesis and characterization of a nanohydroxyapatite/chitosan/polyethylene glycol nanocomposite for bone tissue engineering, Polym. Adv. Technol., 2015, 26, 41-48, DOI: $10.1002 /$ pat.3417.

26 S. Besbes, C. Blecker, C. Deroanne, G. Lognay, N. Drira and $\mathrm{H}$. Attia, Heating effects on some quality characteristics of date seed oil, Food Chem., 2005, 91, 469-476, DOI: 10.1016/ j.foodchem.2004.04.037.

27 P. K. Vayalil, Antioxidant and Antimutagenic Properties of Aqueous Extract of Date Fruit (Phoenix dactylifera L. Arecaceae), J. Agric. Food Chem., 2002, 50(3), 610-617, DOI: 10.1021/jf010716t.

28 M. Dash, S. K. Samal, C. Bartoli, A. Morelli, P. F. Smet, P. Dubruel and F. Chiellini, Biofunctionalization of Ulvan Scaffolds for Bone Tissue Engineering, ACS Appl. Mater. Interfaces, 2014, 6, 3211-3218, DOI: 10.1021/am404912c.

29 M. Shakir, R. Jolly., A. Ahmed, S. S. Ahmed., S. Alam, M. A. Rauf, M. Owais and M. A. Farooqi, Resol based chitosan/nano-hydroxyapatite nanoensernble for effective bone tissue engineering, Carbohydr. Polym., 2018, 179, 317-327, DOI: 10.1016/j.carbpoI.2017.09.103.

30 A. Konermann, M. Staubwasser, C. Dirk, L. Keilig, C. Bourauel, W. Gotz, A. Jager and C. Reichert, Bone substitute material composition and morphology differentially modulate calcium and phosphate release through osteoclast-like cells, Int. J. Oral Maxillofac. Surg., 2014, 43, 514-521, DOI: 10.1016/j.ijom.2013.10.017.

31 X. Cai, H. Tong, X. Shen, W. Chen, J. Yan and J. Hu, Preparation and characterization of homogeneous 
chitosan-polylactic acid/hydroxyapatite nanocomposite for bone tissue engineering and evaluation of its mechanical properties, Acta Biomater., 2009, 5(7), 2693-2703, DOI: 10.1016/j.actbio.2009.03.005.

32 Z. Chen, S. Cao, H. Wang, Y. Li, A. Kishen, X. Deng, X. Yang, Y. Wang, C. Cong, H. Wang and X. Zhang, Biomimetic Remineralization of Demineralized Dentine Using Scaffold of CMC/ACP Nanocomplexes in an In Vitro Tooth Model of Deep Caries, PLoS One, 2015, 10(1), 1-19, DOI: 10.1371/ journal.pone.0116553.

33 S. S. Vaghani, M. M. Patel, C. S. Satish, K. M. Patel and N. P. Jivani, Synthesis and characterization of carboxymethyl chitosan hydrogel: Application as site specific delivery for lercanidipine hydrochloride, Bull. Mater. Sci., 2012, 35(7), 1133-1142.

34 T. V. J. Charpentier, A. Neville, J. L. Lanigan, R. Barker, M. J. Smith and T. Richardson, Preparation of Magnetic Carboxymethylchitosan Nanoparticles for Adsorption of Heavy Metal Ions, ACS Omega, 2016, 1, 77-83, DOI: 10.1021/acsomega.6b00035.

35 N. A. Alomair and H. H. Mohamed, Single step green synthesis of $\mathrm{Au} / \mathrm{ZnO} / \mathrm{rGO}$ nanocomposites for visible light photocatalytic application, Mater. Res. Express, 2019, 6, 1-10, DOI: 10.1088/2053-1591/ab4967.

36 Z. Yu, C. Xiao, Y. Huang, M. Chen, W. Wei, X. Yang, H. Zhou, X. Bi, L. Lu, J. Ruan and X. Fan, Enhanced bioactivity and osteoinductivity of carboxymethyl chitosan/ nanohydroxyapatite/ graphene oxide nanocomposites, $R S C$ Adv., 2018, 8, 17860-17877, DOI: 10.1039/c8ra00383a.

37 M. Shakir, R. Jolly, M. Shoeb and H. M. Khan, Nanohydroxyapatite/chitosan-starch nanocomposite as a novel bone construct: synthesis and in vitro studies, Int. J. Biol. Macromol., 2015, 80, 282-292, DOI: 10.1016/ j.ijbiomac.2015.05.009.

38 P. Moganavally, M. Deepa, P. N. Sudha and R. Suresh, Adsorptive Removal of Lead and Cadmium Ions using Cross-Linked CMC Schiff Base: Isotherm, Kinetics and Catalytic Activity, Orient. J. Chem., 2016, 32(1), 441-453, DOI: $10.13005 / \mathrm{ojc} / 320150$.

39 A. Nabili, A. Fattoum., R. Passas and E. Elalout, Extraction and characrerization of cellulose from date palm cellulose (Phoenix dactylifera L.), Chem. Tech., 2016, 50, 1015-1023.

40 R. Kamel, N. A. El-Wakil, A. F. A. Abdelkhalek and N. A. Elkasabgy, Nanofibrillated cellulose/cyclodextrin based 3D scaffolds loaded with raloxifene hydrochloride for bone regeneration, Int. J. Biol. Macromol., 2020, 156, 704-716, DOI: 10.1016/j.ijbiomac.2020.04.019.

41 S. Saska, H. S. Barud, A. M. M. Gaspar, R. Marchetto, S. J. L. Ribeiro and Y. Messaddeq, Bacterial CelluloseHydroxyapatite Nanocomposites for Bone Regeneration, Int. J. Biomater., 2011, 1-8, DOI: 10.1155/2011/175362.

42 M. Shakir, S. Mirza, R. Jolly, A. Rauf and M. Owais, Synthesis, characterization and in vitro screening of a nanohydroxyapatite/chitosan/Euryale ferox nanoensemble - an inimitable approach for bone tissue engineering, New J. Chem., 2018, 42, 363-371, DOI: 10.1002/jbm.b.31983.
43 N. Sagar, V. P. Soni and J. R. Bellare, Influence of carboxymethyl chitin on stability and biocompatibiliry of 3D nanohydroxyapatite/gelatin/carboxymethyl chitin composite for bone tissue engineering, J. Biomed. Mater. Res., Part $B, 2012$, 100, 624-636, DOI: 10.1002/jbm.b.31983.

$44 \mathrm{M}$. Al-Farsi and C. Y. Lee, Optimization of phenolics and dietary fibre extraction from date seeds, Food Chem., 2008, 108, 977-985, DOI: 10.1016/j.foodchem.2007.12.009.

45 S. Kalliola, E. Repo, V. Srivastava, F. Zhao, J. P. Heiskanen, J. A. Sirvio, H. Liimatainen and M. Sillanpa, Carboxymethyl Chitosan and Its Hydrophobically Modified Derivative as pH-Switchable Emulsifiers, Langmuir, 2018, 34, 2800-2806, DOI: 10.1021/acs.langmuir.7b03959.

46 A. Qidwai, R. Kumar and A. Dikshit, Green synthesis of silver nanoparticles by seed of Phoenix sylvestris L. and their role in the management of cosmetics embarrassment, Green Chem. Lett. Rev., 2018, 11(2), 176-188, DOI: 10.1080/ 17518253.2018.1445301.

47 M. He, C. Chang, N. Peng and L. Zhang, Structure and properties of hydroxyapatite/cellulose nanocomposite films, Carbohydr. Polym., 2012, 87, 2512-2518, DOI: 10.1016/ j.carbpol.2011.11.029.

48 H. Jiang, Y. Zuo, Q. Zou, H. Wang, J. Du, Y. Li and X. Yang, Biomimetic spiral-cylindrical scaffold based on hybrid chitosan/cellulose/nano-hydroxyapatite membrane for bone regeneration, ACS Appl. Mater. Interfaces, 2013, 5, 12036-12044, DOI: 10.1021/am4038432.

49 S. Dayarian, A. Zamani, A. Moheb and M. Maso, PhysicoMechanical Properties of Films of Chitosan, Carboxymethyl Chitosan, and Their Blends, J. Polym. Environ., 2014, 22, 409-416, DOI: 10.1007/s10924-014-0672-9.

50 R. Victor, A. M. Santos, B. V. Sousa, G. A. Neves, L. N. Santana and R. R. Menezes, A Review on Chitosan's Uses as Biomaterial: Tissue Engineering, Drug Delivery Systems and Cancer Treatment, Materials, 2020, 13, 1-71, DOI: 10.3390/ma13214995.

51 K. Kepa, R. Coleman and L. Grøndahl, In vitro mineralization of functional polymers, Biosur. Biotribol., 2015, 1, 214-227, DOI: 10.1016/j.bsbt.2015.09.001.

52 A. Perez-Moreno, M. Reyes-Peces, D. Santos, G. PinagliaTobaruela, E. Orden, J. Vilches-Pérez, M. Salido, M. Piñero and N. Rosa-Fox, Hydroxyl Groups Induce Bioactivity in Silica/Chitosan Aerogels Designed for Bone Tissue Engineering. In Vitro Model for the Assessment of Osteoblasts Behavior, Polymers, 2020, 12, 2802, DOI: 10.3390/polym12122802.

53 R. Wang, J. Guo, X. Lin, S. Chen and S. Ma, Influence of molecular weight and concentration of carboxymethyl chitosan on biomimetic mineralization of collagen, RSC Adv., 2020, 10, 12970-12981, DOI: 10.1039/d0ra00999g.

54 O. Djaoudene, V. López, G. Cásedas, F. Les, C. Schisano, M. B. Bey and G. C. Tenore, Phoenix dactylifera L. seeds: a byproduct as a source of bioactive compounds with antioxidant and enzyme inhibitory properties, Food Funct., 2019, 10, 4953-4965, DOI: 10.1039/C9FO01125K.

55 C. Zhang, S. A. Aldosari, P. S. P. V. Vidyasagar, K. M. Nair, M. G. Nair and M. Nair, Antioxidant and anti- inflammatory 
assays confirm bioactive compounds in Ajwa date fruit, J. Agric. Food Chem., 2013, 61, 5834-5840, DOI: 10.1021/ jf401371v.

56 X. Gao, J. Song, P. Ji, X. Zhang, X. Li, X. Xu, M. Wang, S. Zhang, Y. Deng, F. Deng and S. Wei, Polydopamine-Templated Hydroxyapatite Reinforced Polycaprolactone Composite Nanofibers with Enhanced Cytocompatibility and Osteogenesis for Bone Tissue Engineering, ACS Appl. Mater. Interfaces, 2016, 8(5), 3499-3515, DOI: 10.1021/acsami.5b12413.

57 S. Sirisena, K. Ng and S. Ajlouni, The Emerging Australian Date Palm Industry: Date Fruit Nutritional and Bioactive Compounds and Valuable Processing By-Products, Compr. Rev. Food Sci. Food Saf., 2015, 14, 813-823, DOI: 10.1111/ 1541-4337.12162.

58 H. Xie, P. Wang and J. Wu, Effect of exposure of osteoblastlike cells to lowdose silver nanoparticles: uptake, retention and osteogenic activity, Artif. Cells, Nanomed., Biotechnol., 2019, 47(1), 260-267, DOI: 10.1080/21691401.2018.1552594.

59 S. Lepretre, F. Chai, J.-C. Hornez, G. Vermet, C. Neut, M. Descamps, H. F. Hildebrand and B. Martel, Prolonged local antibiotics delivery from hydroxyapatite functionalised with cyclodextrin polymers, Biomaterials, 2009, 30, 6086-6093, DOI: 10.1016/j.biomaterials.2009.07.045.

60 L. Tao, L. Zhonglong, X. Ming, Y. Zezheng, L. Zhiyuan, Z. Xiaojun and W. Jinwu, In vitro and in vivo studies of a gelatin/carboxymethyl chitosan/LAPONITE ${ }^{\circledR}$ composite scaffold for bone tissue engineering, RSC Adv., 2017, 7, 54100-54110, DOI: 10.1039/c7ra06913h.

61 S. A. Umoren, Z. M. Gasem and I. B. Obot, Natural Products for Material Protection: Inhibition of Mild Steel Corrosion by Date Palm Seed Extracts in Acidic Media, Ind. Eng. Chem. Res., 2013, 52, 14855-14865, DOI: 10.1021/ie401737u.

62 L. L. Miranda, V. P. Guimarães-Lopes, L. S. Altoé, M. M. Sarandy, F. C. S. A. Melo, R. D. Novaes and R. V. Gonçalves, Plant Extracts in the Bone Repair Process: A Systematic Review, Mediators Inflammation, 2019, 1-22, DOI: $10.1155 / 2019 / 1296153$.

63 M. M. Islam, M. Shahruzzaman, S. Biswas, M. Nurus Sakib and T. U. Rashid, Chitosan based bioactive materials in tissue engineering applications-A review, Bioact. Mater., 2020, 5, 164-183, DOI: 10.1016/j.bioactmat.2020.01.012.

64 J. R. Porter, T. T. Ruckh and K. C. Popat, Bone Tissue Engineering: A Review in Bone Biomimetics and Drug Delivery Strategies, Biotechnol. Prog., 2009, 25(6), 1539-1560, DOI: 10.1002/btpr.246.

65 I. Zia, R. Jolly, S. Mirza, M. S. Umar, M. Owais and M. Shakir, Hydroxyapatite nano-particles fortified Xanthan gum - Chitosan based polyelectrolyte complex scaffolds for supporting osteo-friendly environment, ACS Appl. Bio Mater., 2020, 3(10), 7133-7146, DOI: 10.1021/acsabm.0c00948permi. 